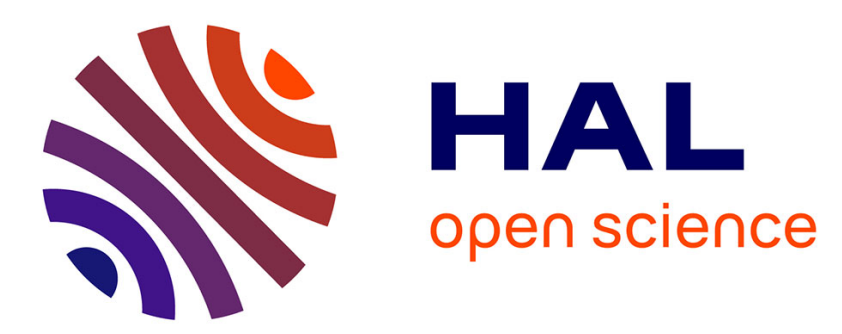

\title{
Unravelling the Anomalous Dielectric Permittivity of Nanoconfined Electrolyte Solutions
}

\author{
Richard Renou, Anthony Szymczyk, Aziz Ghoufi
}

\section{To cite this version:}

Richard Renou, Anthony Szymczyk, Aziz Ghoufi. Unravelling the Anomalous Dielectric Permittivity of Nanoconfined Electrolyte Solutions. Nanoscale, 2015, 7 (15), pp.6661-6666. 10.1039/C5NR00508F . hal-01132817

\section{HAL Id: hal-01132817 https://hal-univ-rennes1.archives-ouvertes.fr/hal-01132817}

Submitted on 29 Oct 2015

HAL is a multi-disciplinary open access archive for the deposit and dissemination of scientific research documents, whether they are published or not. The documents may come from teaching and research institutions in France or abroad, or from public or private research centers.
L'archive ouverte pluridisciplinaire HAL, est destinée au dépôt et à la diffusion de documents scientifiques de niveau recherche, publiés ou non, émanant des établissements d'enseignement et de recherche français ou étrangers, des laboratoires publics ou privés. 


\title{
Unravelling the Anomalous Dielectric Permittivity of Nanoconfined Electrolyte Solutions.
}

\author{
Richard Renou $^{a}$, Anthony Szymczyk ${ }^{a}$ and Aziz Ghoufi ${ }^{*} b$
}

The dielectric properties of sodium chloride solutions confined in a hydrophilic nanocavity were investigated by means of molecular dynamics simulations. Unlike what is observed in the bulk phase, three dielectric regimes were evidenced, namely an anomalous increase in the dielectric permittivity at low concentrations (with respect to confined pure water), a dielectric plateau at intermediate concentrations and finally a bulk-like behavior for salt concentrations higher than a critical value. It was shown that this peculiar behavior results from the competition between dielectric saturation due to the electric field generated by ions (which tends to lower the dielectric permittivity) and the ion-induced perturbation of pre-oriented water molecules inside the nanocavity which gain some rotational degrees of freedom (entropic contribution) leading to an increase in dipolar fluctuations responsible for the increase in the dielectric permittivity.

\section{Introduction}

The decrease in the dielectric constant $\varepsilon$ of water with increasing electrolyte concentration in bulk phase has been first observed in $1894^{1}$. Since, many works were devoted to the understanding of this phenomenon ${ }^{2-6}$. Thus, it was shown that this dielectric behavior results from the dipolar saturation i.e. the screening of solvent dipoles by ions leading to a decrease in dipole-dipole correlations ${ }^{7,8}$. Actually, the dielectric permittivity of a solvent measures the ability of molecules to orientate themselves in the direction of an applied external electrical field. In the presence of electrolytes, water molecules are locally oriented around ions in the first and second hydration shells, thus preventing them to orientate themselves along the external electrical field. This local pre-orientation therefore leads to the decrease in $\varepsilon$ as the electrolyte concentration increases. In contrast with the bulk phase, the dielectric behavior of electrolyte solutions confined at the nanoscale was less studied despite the raise of nano-applications such as desalination by nanoporous membranes. Nonetheless, an anomalous dielectric behavior of $\mathrm{NaCl}$ solutions confined into a cylindrical silica nanotube was recently reported from molecular dynamics simulations ${ }^{9,10}$. In a cylindrical nanotube the dielectric permittivity is a local diagonal tensor such that both radial $\left(\varepsilon_{\perp}\right)$ and axial $\left(\varepsilon_{\|}\right)$components can be defined. Despite the

a Institut des Sciences Chimiques de Rennes, UMR 6226 CNRS, Université de Rennes 1, 263 Avenue du Général Leclerc, 35042 Rennes, France; Université Européenne de Bretagne, France

${ }^{b}$ Institut de Physique de Rennes, UMR CNRS 6251, Université Rennes 1, 263 avenue du Général Leclerc, 35042 Rennes, *E-mail: aziz.ghoufi@univrennes1.fr ambiguity on the calculation of $\varepsilon_{\perp}{ }^{11,12}$ a dielectric anisotropy between both components was reported, which was recovered in recent numerical and experimental studies ${ }^{13-16}$. Furthermore, under cylindrical confinement it was highlighted that $\varepsilon_{\|}$ decreased as a function of $\mathrm{NaCl}$ concentration $\left(\mathrm{c}_{\mathrm{NaCl}}\right)$, which corresponds to a bulk-like behavior, while $\varepsilon_{\perp}$ was found to increase up to a critical concentration about $0.5 \mathrm{~mol}$. $\mathrm{L}^{-1}$ $\left(c_{L}\right)$. Beyond $c_{L}$ a bulk-like behavior was recovered i.e. a decrease in $\varepsilon_{\perp}$ for increasing $\mathrm{c}_{\mathrm{NaCl}}{ }^{9,10}$. It was shown that this anomalous increase in the dielectric permittivity resulted from the rapid hydrogen bonds (HBs) rearrangement of water molecules so as to maintain their HB network. Indeed, if the ion concentration is not too high, the degree of disturbance of the HB network induced by the presence of ions remains limited and water molecules succeed in rearranging themselves around ions while maintaining their HB network. It was observed that this reorganization of water molecules led to an increase in the fluctuations of water dipoles, thus resulting in the increase in the dielectric permittivity. However, the microscopic mechanism responsible for this peculiar dielectric behaviour, specific to confined electrolyte solutions, is not fully understood and the present work aims at providing insight into its molecular origin. Thanks to molecular dynamics (MD) simulations of sodium chloride solutions confined in a spherical nanocavity, we show that the increase in the dielectric permittivity with $\mathrm{NaCl}$ concentration (up to a critical concentration) is related to the disruption of the initial pre-ordering of water molecules inside the nanocavity (due to confinement effect) induced by the presence of ions. Let us note that in a spherical cavity the calculation of $\varepsilon$ is rigorous and unambiguous ${ }^{17}$. Additionally, this $3 \mathrm{D}$ confinement allows us to well 
capture the effects of confinement on the dielectric permittivity.

\section{Computational Details}

The spherical silica nanocavity was build by carving a sphere of diameter around $24 \AA$ (see Figure 1) in an amorphous silica block. The method of Brodka and Zerda was used ${ }^{18}$. This approach allows us to build a hydrophilic cavity with a silanol density of $7.5 \mathrm{SiOH} / \mathrm{nm}^{2}$ corresponding to a highly hydrated protonated silica surface ${ }^{18,19}$. Details on the construction of the nanoporous framework and the force field can be found elsewhere ${ }^{12,20-23}$. The sides of the cubic simulation box were $L_{x}=L_{y}=L_{z}=35 \AA$. The silica force field was taken from Refs. ${ }^{12,24}$. Although the silica matrix was subsequently kept rigid, the rotation around the $\mathrm{Si}-\mathrm{O}$ bond of the hydroxyl groups was allowed by means of a bending potential between $\mathrm{Si}, \mathrm{O}$ and $\mathrm{H}$. Ions were considered as unpolarizable $^{25}$ and water molecules were modeled by means of the rigid TIP4P/2005 model ${ }^{26}$. Indeed, we showed that the polarizability of $\mathrm{Na}^{+}$and $\mathrm{Cl}^{-}$ions had a negligible impact on both their structure and dynamics properties ${ }^{12,24}$. The density of confined water was calculated by means of hybrid Grand Canonical Monte Carlo (GCMC)/Molecular dynamics (MD) simulations (GCMC/MD) where the number of $\mathrm{Na}^{+}$and $\mathrm{Cl}^{-}$ ions was kept constant and the absence of ion-pair clusters was checked. The number of water molecules was calculated by means of the insertion/deletion MC move. Thus, the number of water molecules was adjusted as a number of ions. 9 concentrations were explored corresponding to a $\mathrm{NaCl}$ number, $\mathrm{N}_{\mathrm{NaCl}}=0,1,2,3,4,5,6,9,20$. The corresponding numbers of water molecules were 282, 285, 282, 282,285, 277, 278, 275 and $257 . \mathrm{NaCl}$ concentration in the cavity was computed from $\mathrm{C}=\mathrm{N}_{\mathrm{NaCl}} / \mathrm{V}$, where $\mathrm{V}$ is the volume of the cavity. This hybrid method allows modeling ion dynamics and their hydration shell through an MD move into a GCMC scheme ${ }^{27}$. It is an alternative to the cluster MC move for modeling the displacement of the ion hydration shell. For the MC scheme, the ratios for each trial move were defined as follows: 0.1995 for the translation, 0.1995 for the rotation, 0.6 for the insertion/deletion, and 0.001 for the MD move. The MD runs corresponded to 5000 steps $\left(N_{M D}\right)$ using a time step of 0.001 ps $\left(t_{M D}\right)$. We considered more than 70000 GCMC/MD cycles. To validate our findings, several ratios for each trial move and $\left(\mathrm{N}_{M D}, \mathrm{t}_{M D}\right)$ couples were tested. The chemical potential $(\mu)$ of water molecules was $2431 K$ (corresponding to a pressure of 1 bar). The chemical potential of water at $300 \mathrm{~K}$ and 1 bar was calculated from the Widom insertion method ${ }^{28}$. MD simulations were performed using a time step of $0.001 \mathrm{ps}$ to sample $30 \mathrm{~ns}$ (acquisition phase). The equilibration time was $10 \mathrm{~ns}$. All MD simulations were carried out with the DL_POLY package $^{29}$ using a combination of the velocity-Verlet, QUATER-
NION, SHAKE-RATTLE algorithms ${ }^{30}$ and the Nose-Hoover thermostat ${ }^{31,32}$ with a relaxation time of $\tau_{t}=0.5 \mathrm{ps}$. Periodic boundary conditions were applied in the three directions. MD simulations were performed in the NVT statistical ensemble at $\mathrm{T}=300 \mathrm{~K}$. The Ewald summation was used for calculating electrostatic interactions and short-ranged interactions were truncated at $12 \AA$. The local dielectric permittivity was computed by using the relation derived by Blaak and Hansen ${ }^{17}$ $\varepsilon_{r}(r)=1+\beta \varepsilon_{0}^{-1}\left[\langle\mathbf{m}(\mathbf{r}) \mathbf{M}\rangle_{0}-\langle\mathbf{m}(\mathbf{r})\rangle_{0}\langle\mathbf{M}\rangle_{0}\right]$, where $\mathbf{m}(\mathbf{r})$ is the polarization density such as $\mathbf{M}=\int_{V} \mathbf{m}(\mathbf{r}) d \mathbf{r}, \mathbf{M}$ is the total dipole moment, $\langle\ldots\rangle_{0}$ denotes a statistical average over the different configurations, $\beta$ is the inverse temperature and $\varepsilon_{0}$ the vacuum permittivity. The local components of the permittivity were computed from the dipole moments of water molecules and ion pairs. Let us note that in a confined spherical medium the dielectric permittivity is a tensor and then an overall dielectric constant cannot be calculated.

\section{Results and Discussion}

We report in Figure 2a the radial profile of the dielectric permittivity of $\mathrm{NaCl}$ solutions as a function of $\mathrm{NaCl}$ concentration $\left(\mathrm{c}_{\mathrm{NaCl}}\right)$. As shown in Figure $2 \mathrm{a}$ the dielectric constant of the pure confined water is lower than that of the bulk phase. Moreover, Figure 2a depicts the increase in the dielectric permittivity with $\mathrm{NaCl}$ concentration. Let us note that the rise in $\varepsilon$ takes place from the centre of the cavity to the cavity wall. Figure $2 b$ shows the radially averaged value of the dielectric constant and highlights three characteristic zones i) from 0.0 mol. $\mathrm{L}^{-1}$ to $0.7 \mathrm{~mol} . \mathrm{L}^{-1}$ where the dielectric permittivity increases with $\mathrm{c}_{\mathrm{NaCl}}$, ii) a second region between 0.7 mol. $\mathrm{L}^{-1}$ and 2 mol. $\mathrm{L}^{-1}$ corresponding to a plateau, and iii) a third zone where $\varepsilon$ decreases as $\mathrm{c}_{\mathrm{NaCl}}$ increases. This dielectric behavior is in line with the results obtained by confining $\mathrm{NaCl}$ solutions in a silica nanotube ${ }^{9}$ but is different from what is observed in the bulk phase (see Figure $2 b$ ). If the structural origin of the decrease in $\varepsilon$ in bulk phase is well known (dipolar saturation) the molecular mechanism responsible for the increase in $\varepsilon$ under confinement remains to elucidate.

The dielectric permittivity of confined liquids is impacted by excluded volume effects. In order to explore the correlation between the local density and the dielectric permittivity we report in Figure $3 \mathrm{a}$ the radial profile of water density as a function of $\mathrm{NaCl}$ concentration. Four water layers are found inside the nanocavity, whatever $\mathrm{c}_{\mathrm{NaCl}}$ and the local density of water is barely impacted by the presence of ions. Moreover, as evidenced in Figure 3b, ions are present in three of the four water layers and are absent from the interfacial layer. Ions are not located in the close vicinity of the surface so as to preserve their hydration shell, thus avoiding the high energy 
penalty associated with dehydration. These results indicate that the radial organization of ions is not responsible for the dielectric behavior reported in Figure 2 since the increase in $\varepsilon$ takes place from the centre of the cavity to the cavity wall.

As discussed in the introduction, the dielectric constant is a measure of the ability of molecules to orientate themselves in response to the application of an electric field. Therefore, the dielectric permittivity of an aqueous solution is directly impacted by the orientation of water molecules. Under confinement, the dielectric behavior of water is connected to the orientation of water molecules with respect to the confining surface. We report in Figure 4a the angular distribution of the angle between the dipole moment vector of water molecules and the (outgoing) surface normal vector. Two regions were distinguished; the interfacial region between $r=8 \AA$ and $r=12 \AA$ and the central region between $r=0 \AA$ and $r=8$ $\AA$ ( $r=0 \AA$ corresponds to the centre of the cavity). As shown in Figure 4a, for pure water a strong orientation of water molecules is observed. Indeed, the maxima of the angular distributions are found between $0^{\circ}$ and $60^{\circ}$ and between $0^{\circ}$ and $50^{\circ}$ for water molecules in the vicinity of the cavity wall and in the central part of the cavity, respectively. This pre-orientation (due to both excluded volume effects and interactions with the surface) makes it more difficult the orientation of water molecules in the direction of an applied external electrical field, which explains the decrease in $\varepsilon$ of pure water inside the nanocavity with respect to the bulk value. HBs formed between $\mathrm{SiOH}$ surface groups and interfacial water molecules contribute to the pre-orientation of these latter. We found $0.8 \mathrm{HB}$ (per water molecule) between $\mathrm{SiOH}$ groups and water molecules, i.e. $20 \%$ of the total HBs $(\sim 3.9$ per water molecule). This interfacial hydrophilic anchoring also impacts the orientation of water molecules in the water layers farther from the surface (HB network between water layers), which explains that a preferential orientation is also observed in the central part of the cavity (although it is less marked than in the interfacial region; see Figure 4a). An illustration of this pre-orientation is given in Figure 5a. In a future work it would be interesting to explore the dielectric behavior of water confined in a nanocavities with hydrophobic surface properties.

When electrolytes are introduced within the nanocavity, a modification of water molecule organization is expected. However, as shown in Figure 3 a the layering organization remains almost unchanged with only a slight decrease in the local density of water. Moreover, HBs between water molecules and $\mathrm{SiOH}$ surface groups are found to be almost independent of $\mathrm{NaCl}$ concentration. Indeed, we found $0.8 \mathrm{HB}$ (per water molecule) between $\mathrm{SiOH}$ groups and water molecules, whatever $\mathrm{c}_{\mathrm{NaCl}}$. Therefore, HBs formed between water molecules and the surface are not responsible for the modification of the dielectric permittivity reported in Figure 2a. On the other hand, as depicted in Figure $4 \mathrm{~b}$ the orientation of water molecules is drastically modified at the central part of the cavity, i.e. where ions are located (see Figure 3a). The initial pre-orientation of water molecules induced by the surface (see Figure 5a) is progressively lost as the electrolyte concentration increases. Indeed, the profiles of angular distribution become flatter as $\mathrm{c}_{\mathrm{NaCl}}$ increases from $0.2 \mathrm{~mol} . \mathrm{L}^{-1}$ to $0.9 \mathrm{~mol} . \mathrm{L}^{-1}$ (see Figure $4 \mathrm{~b}$ ). The angular and the dielectric distributions are clearly correlated since the three previously defined zones (Figure 2b) can be also observed through the angular distributions. It seems that the increase in $\varepsilon$ is due to ions that disturb the local orientation of water molecules. This structural perturbation provides an increase in the degrees of freedom of water molecules inducing an increase in dipolar fluctuations. This can be correlated to an increase in disorder and then to an entropic contribution of the increase in $\varepsilon$. The plateau observed in Figure $2 b$ for intermediate values of $\mathrm{c}_{\mathrm{NaCl}}$ results from the competition between this entropic contribution and the dielectric saturation phenomenon (i.e. the orientation of water dipoles around ions). The decrease in $\varepsilon$ for $\mathrm{c}_{\mathrm{NaCl}}$ higher than 2 mol. $\mathrm{L}^{-1}$ results from dielectric saturation that becomes the dominant mechanism as $\mathrm{c}_{\mathrm{NaCl}}$ exceeds a critical value ( an illustration of the orientation of water molecules around ions is given in Figure 5b). There is therefore a competition between the dipolar saturation due to the electric field generated by ions, leading to a decrease in the dielectric permittivity as the $\mathrm{NaCl}$ concentration increases, and the ion-induced perturbation of the pre-oriented water molecules which gain some rotational degrees of freedom (entropic contribution) leading to an increase in the dipolar fluctuations and hence, to an increase in the dielectric permittivity. This second phenomenon increases until the ion concentration becomes high enough so that the dipolar saturation effect becomes dominant, thus reducing the dielectric permittivity of the confined electrolyte solution (bulk-like behaviour). The dielectric behaviour of confined electrolyte solutions therefore depends on the amount of ions in the cavity. Dipolar saturation is linked to the strong orientation of water molecules around the ions. Indeed, by increasing ion concentration the number of oriented water molecules increases inducing a decrease in the dielectric permittivity. The coordination number of $\mathrm{Na}^{+}$and $\mathrm{Cl}^{-}$ was found to be 5.4 and 8.1, respectively. It was calculated in the first hydration shell. The distance allowing us to define the first hydration sphere was inferred from the radial distribution functions between ions and the centre of mass of water molecules. Distances of 3.4 and $4.2 \AA$ were found for $\mathrm{Na}^{+}$ and $\mathrm{Cl}^{-}$, respectively. These results are in good agreement with those provided in Ref. ${ }^{25}$. It can be noted that the coordination numbers of both ions were found identical for all $\mathrm{c}_{\mathrm{NaCl}}$. Therefore the number of oriented water molecules increased 
gradually as a function of $\mathrm{NaCl}$ concentration. As mentioned above, the pre-orientation of solvent molecules largely contributes to the decrease in the dielectric permittivity. This preorientation around ions has been quantified by calculating the angular distribution of the angle between the dipole moment of water molecules and the vector between ions and the centre of mass of water molecules (molecules in both the first and second hydration shells were considered). As expected, water molecules exhibit preferential orientation around both kinds of ions as shown in Figure 6. For $\mathrm{Na}^{+}$and $\mathrm{Cl}^{-}$the maximum probability was found around $178^{\circ}$ and $50^{\circ}$, respectively. Figure 6 shows that the structure of water around $\mathrm{Na}^{+}$ions is similar inside the nanocavity and in the bulk phase. On the other hand, $\mathrm{Cl}^{-}$seems to be more sensitive to confinement effects, the angular distribution of water molecules around $\mathrm{Cl}^{-}$being sharper inside the nanocavity than in the bulk phase. That results from geometrical restrictions inside the cavity, $\mathrm{Cl}^{-}$being more impacted than $\mathrm{Na}^{+}$cations because of its larger coordination number. Let us note that the experimental data is all for cylindrical nanopores while our simulations focused on a nanosphere. The so-obtained results are not transferable to a nanotube because in a sphere there is an isotropic confinement while in a nanotube the confinement is only along the radial direction whereas the axial direction is unconfined.

\section{Concluding Remarks}

To summarize, we predicted the radial component of the dielectric constant of $\mathrm{NaCl}$ solutions confined in a spherical nanocavity of diameter around $24 \AA$. Unlike what is observed in the bulk phase, three dielectric regimes were evidenced i) an anomalous increase in $\varepsilon$ at low concentrations (from 0.0 mol. $\mathrm{L}^{-1}$ to $0.7 \mathrm{~mol} . \mathrm{L}^{-1}$ ) ii) a dielectric plateau at intermediate concentrations (between $0.7 \mathrm{~mol} . \mathrm{L}^{-1}$ to $2 \mathrm{~mol} . \mathrm{L}^{-1}$ ) and iii) a decrease in $\varepsilon$ beyond 2.0 mol.L $\mathrm{L}^{-1}$. This dielectric behavior is different from what is observed in the bulk phase where a monotonous decrease in $\varepsilon$ is observed as a function of the salt concentration. This peculiar behavior was explained in terms of the pre-orientation of water molecules. Indeed, in confined media water molecules are pre-oriented because of excluded volume effects and the surface interactions, which explains the lower value of $\varepsilon$ for confined pure water with respect to bulk water. At low salt concentrations, ions disturb this preorientation and water molecules gain orientational degrees of freedom (entropic contribution), which makes it easier the orientation of water molecules in response to an external electrical field. At intermediate salt concentrations, a competition between this entropic contribution and the dipolar saturation leads a dielectric plateau. Finally, at sufficiently high concentrations the dipolar saturation becomes the dominant mechanism and a bulk-like behavior is recovered, i.e. $\varepsilon$ decreases as the salt concentration increases.

\section{Acknowledgements}

The authors are grateful to the Agence Nationale de la Recherche for its financial support through the program MUTINA (ANR 2011 BS09 002).

\section{References}

1 P. Drude and W. Z. Nernst, Phys. Chem., 1894, 15, 79.

2 R. T. Lattey, Phil. Mag., 1921, 41, 829.

3 C. C. Schmidt, Phys. Rev., 1927, 30, 925.

4 J. B. Hasted, D. M. Riston and C. H. Collie, J. Chem. Phys., 1948, 16, 1.

5 J. Israelachvili and H. Wennerström, Nature, 1996, 379, 219.

6 Y. Marcus, Chem. Rev., 2011, 111, 2761.

7 D. Levesque, J. J. Weis and G. N. Patey, J. Chem. Phys., 1980, 72, 1887.

8 G. N. Patey and S. L. Carnie, J. Chem. Phys., 1983, 78, 5183.

9 H. Zhu, A. Ghoufi, A. Szymczyk, B. Balannec and D. Morineau, Phys. Rev. Lett., 2012, 109, 107801.

10 R. Renou, A. Ghoufi, A. Szymczyk, H. Zhu, J.-C. Neyt and P. Malfreyt, J. Phys. Chem. C, 2013, 117, 11017.

11 S. Gekle and A. Arnold, Phys. Rev. Letters, 2013, 111, 089801 .

12 H. Zhu, A. Ghoufi, A. Szymczyk, B. Balannec and D. Morineau, Phys. Rev. Lett., 2013, 111, 089802.

13 H.-B. Cui, K. Takahashi, Y. Okano, H. Kobayashi, Z. Wang and A. Kobayashi, Angew. Chem., 2005, 117, 6666.

14 Y. Lin, J. Shiomi, S. Maruyama and G. Amberg, Phys. Rev. $B, 2009,80,045419$.

15 F. Mikami, K. Matsuda, H. Kataura and Y. Maniwa, ACS Nano, 2009, 3, 1279.

16 C. Zhang, F. Gygi and G. Galli, J. Phys. Chem. Lett., 2013, 4, 2477.

17 R. Blaak and J.-P. Hansen, J. Chem. Phys., 2006, 124, 144714.

18 A. Bródka and T. W. Zerda, J. Chem. Phys., 1996, 104, 6319.

19 J. Puibasset and R. J.-M. Pellenq, J. Chem. Phys., 2005, 122, 094704.

20 R. Guégan, D. Morineau and C. Alba-Simonesco, Chem. Phys., 2005, 317, 236.

21 R. Busselez, R. Lefort, Q. Ji, F. Affouard and D. Morineau, Phys. Chem. Chem. Phys, 2009, 11, 11127.

22 A. Ghoufi, G. Maurin and G. Férey, J. Phys. Chem. Lett., 2010, 1, 2810. 
23 A. Ghoufi, D. Morineau, R. Lefort, I. Hureau, L. Hennous, H. Zhu, A. Szymczyk, P. Malfreyt and G. Maurin, J. Chem. Phys, 2011, 134, 074104.

24 A. Ghoufi, A. Subercaze, Q. Ma, P. Yot, Y. Ke, I. PuenteOrench, T. Devic, V. Guillerm, C. Zhong, C. Serre, G. Férey and G. Maurin, J. Phys. Chem. C, 2012, 116, 13289.

25 R. G. Moutain, Int. J. Thermophys., 2007, 28, 536.

26 J. Abascal and C. Vega, J. Chem. Phys., 2005, 123, 23505.

27 A. Ghoufi and G. Maurin, J. Phys. Chem. C, 2010, 114, 6496.

28 B. Widom, J. Chem. Phys., 1963, 39, 2808.

29 T. R. Forester and W. Smith, DLPOLY ,CCP5 Program Library, Daresbury Lab., U.K., 1004.

30 M. P. Allen and D. J. Tildesley, Computer Simulations of Liquids, Oxford, 1987.

31 S. Nose, J. Chem. Phys., 1984, 81, 511.

32 W. Hoover, Phys. Rev. A, 1985, 31, 1695. 


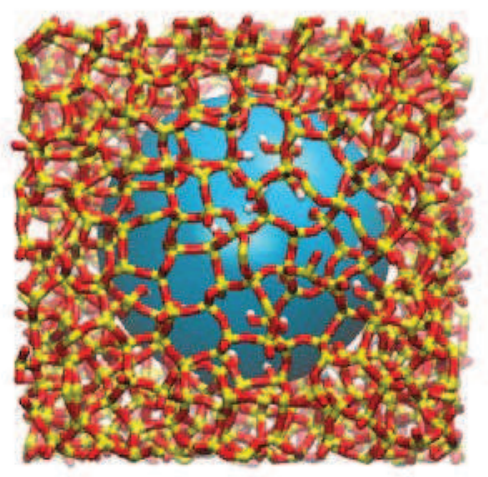

Fig. 1 Illustration of a spherical nanocavity (in cyan) carved in a silica cubic block. Red, yellow and white atoms correspond to oxygen, silicon and hydrogen atoms, respectively. 

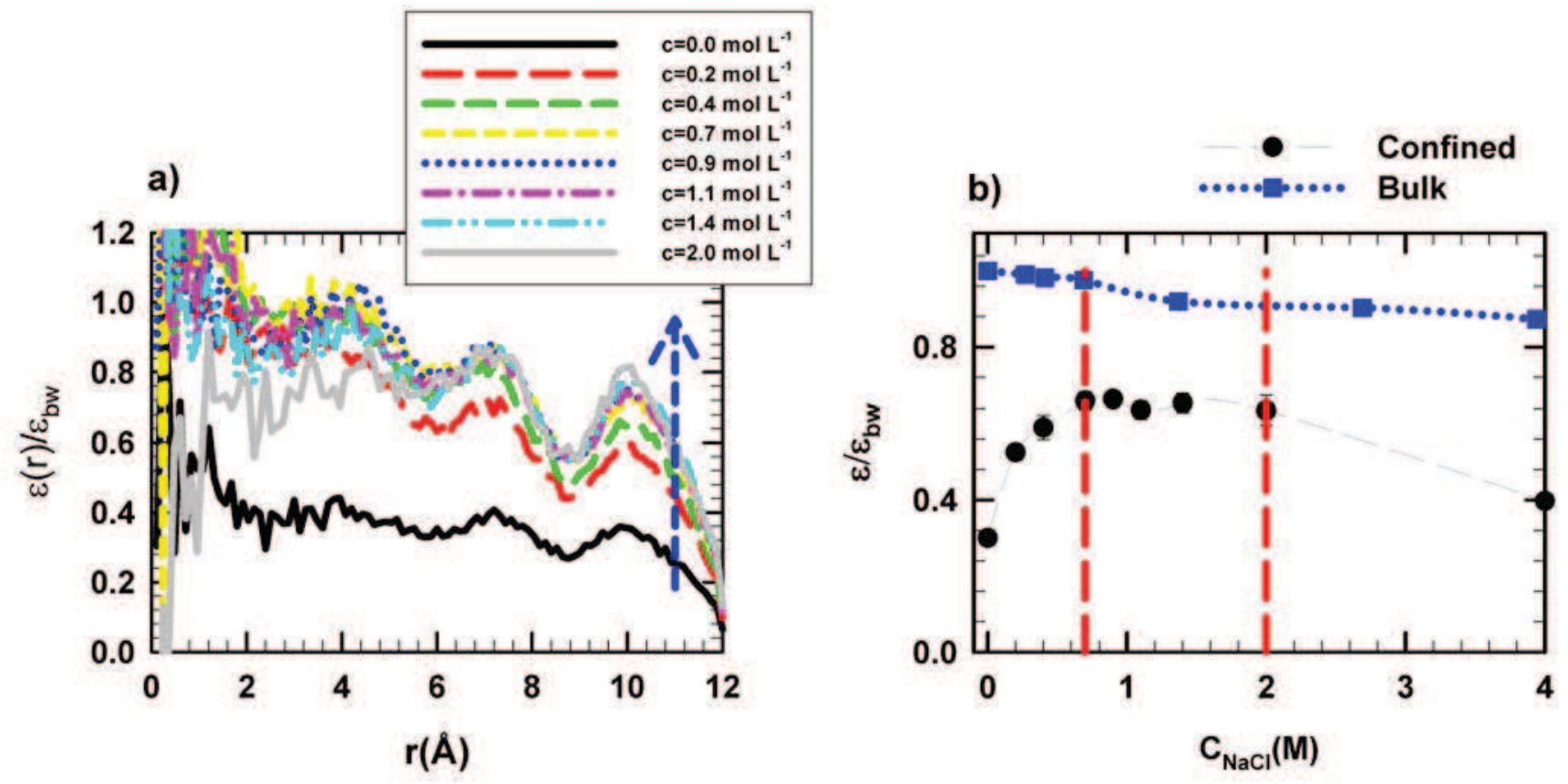

Fig. 2 a)Radial dielectric permittivity as a function of $\mathrm{NaCl}$ concentration. The position $r=0 \AA$ corresponds to the cavity centre. $\varepsilon_{b w}$ corresponds to the dielectric permittivity of pure water in the bulk phase. The vertical arrow is a guide line to show the increase in $\varepsilon$ as a function of $\mathrm{c}_{\mathrm{NaCl}}$. b) Radially averaged value of the dielectric constant as a function of $\mathrm{c}_{\mathrm{NaCl}}$. The vertical red lines indicate the limit of the three dielectric zones i) increase in $\varepsilon$ ii) dielectric plateau and iii) decrease in $\varepsilon$. 

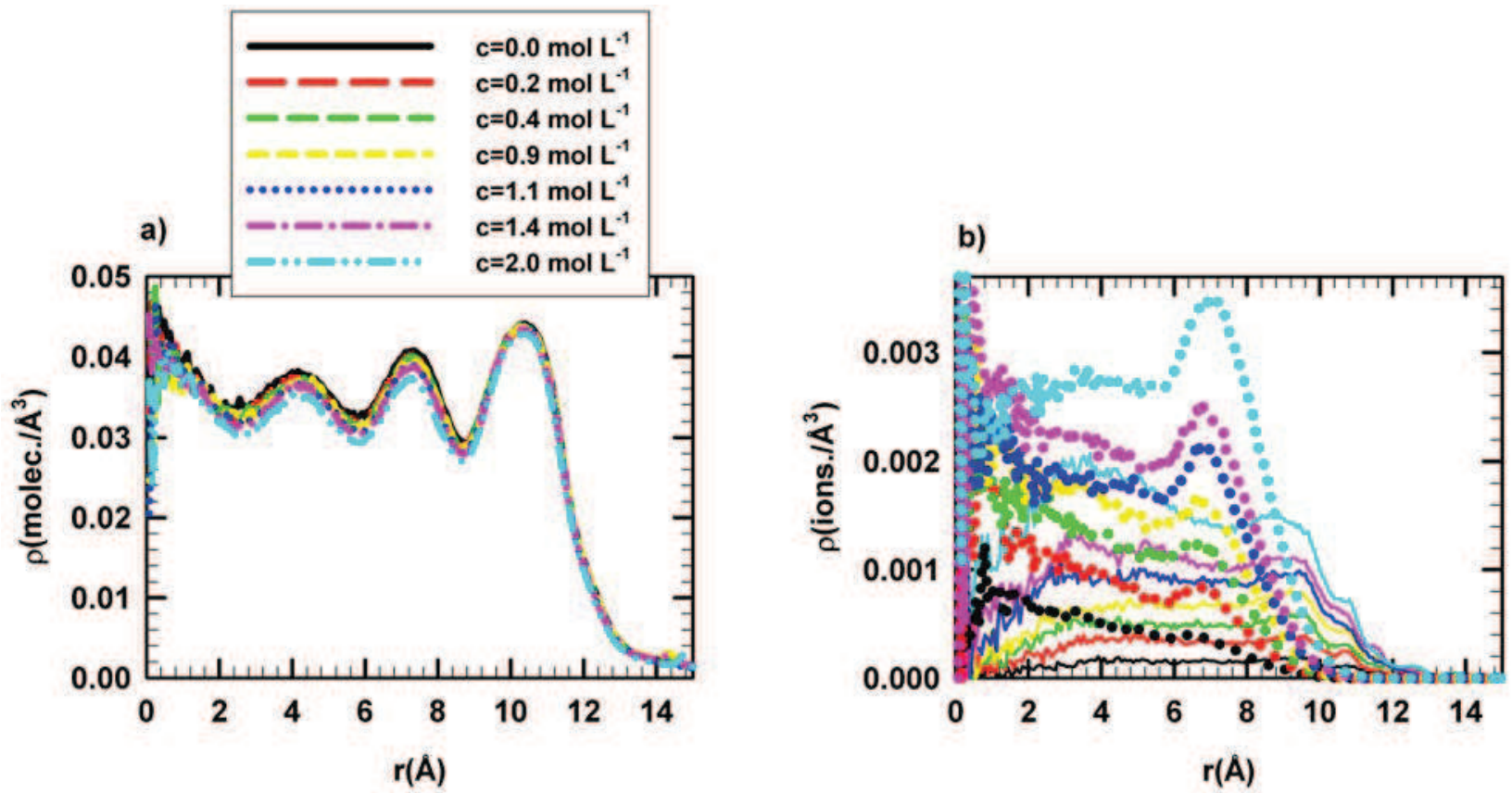

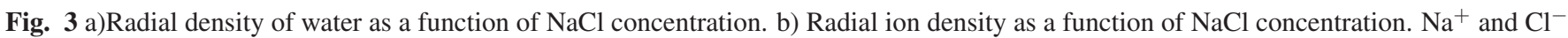
ions are represented in solid and dotted lines, respectively. Color coding is the same as in a). In a) and b) the position $r=0 \AA$ corresponds to the cavity centre. 
a)

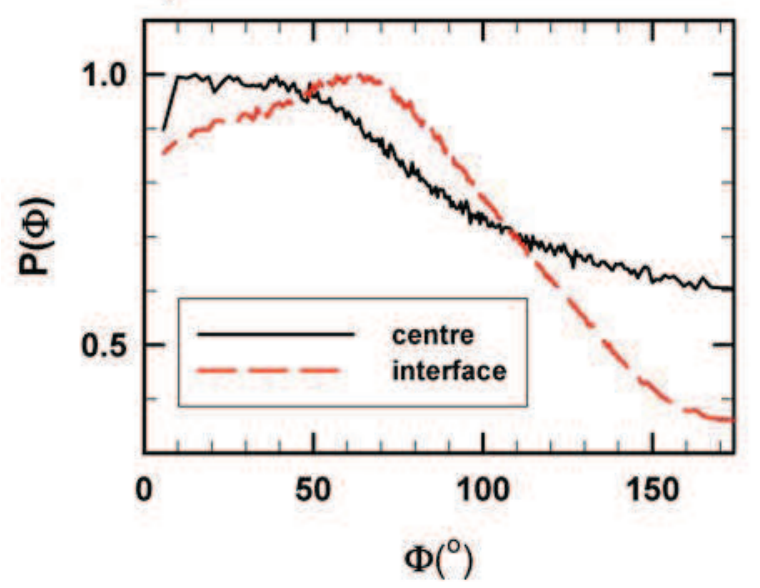

b)

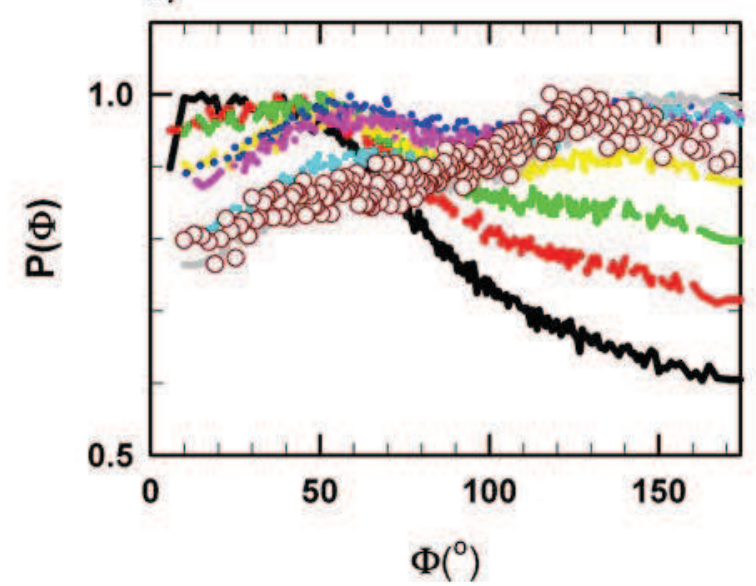

Fig. 4 a)Angular distribution between the dipolar moment of water molecules in the interfacial region and at the centre of the cavity and the outgoing normal vector to the silica surface. b)Angular distribution between the dipolar moment of water molecules at the centre of the cavity and the outgoing normal vector to the surface as a function of $\mathrm{NaCl}$ concentration. Color coding is the same as in Figure 3a. Empty symbols represent the results for $\mathrm{c}=4.0 \mathrm{~mol} \cdot \mathrm{L}^{-1}$. 

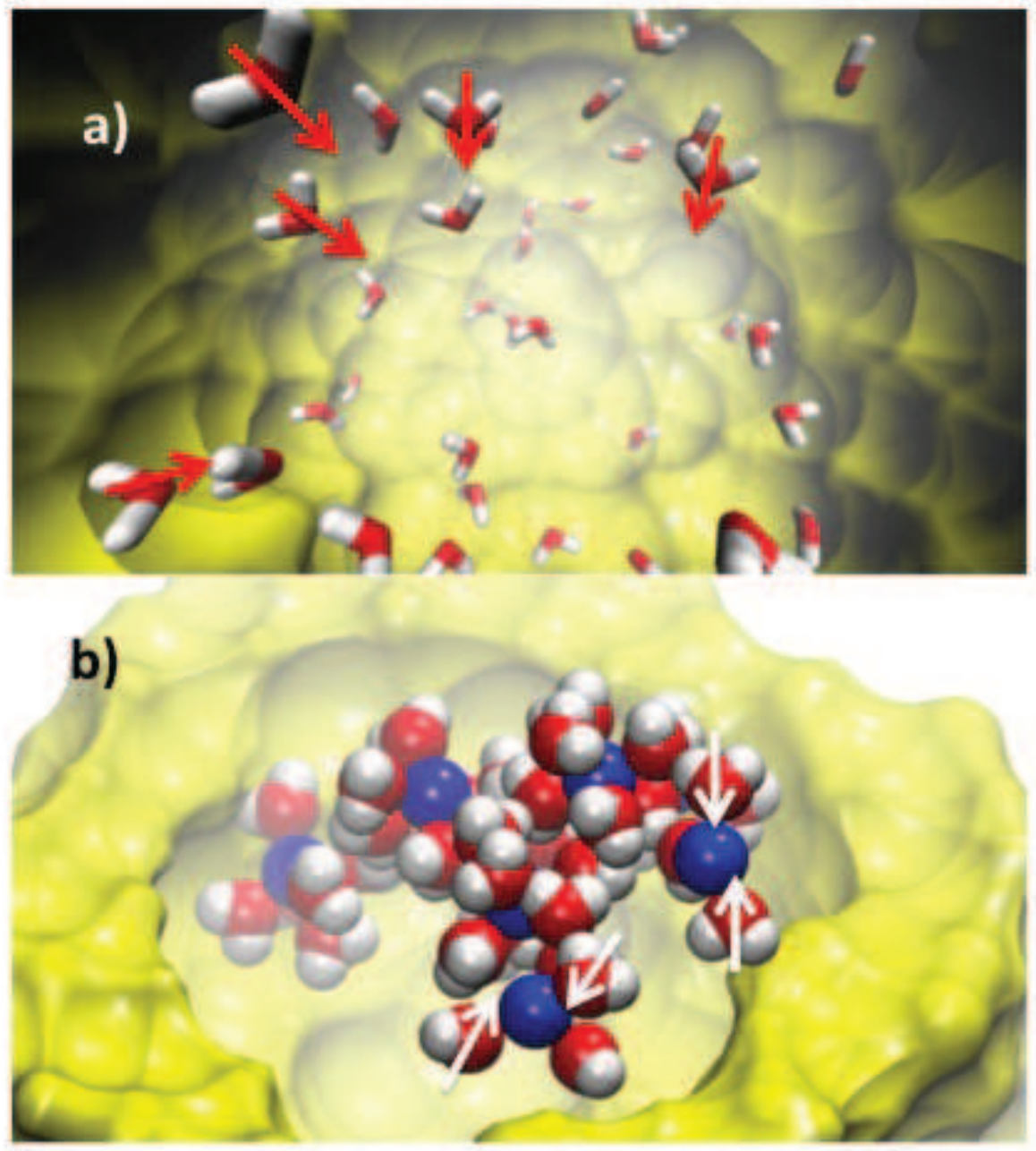

Fig. 5 a) Illustration of pre-oriented water molecules in the nanocavity. The red arrows indicate the orientation of the dipole moment of water molecules. b) Illustration of oriented water molecules around $\mathrm{Na}^{+}$cations. Yellow, red, white and blue colors correspond to the silica matrix, oxygen and hydrogen atoms and $\mathrm{Na}^{+}$ions. The white arrows indicate the orientation of the dipole moment of water molecules. 


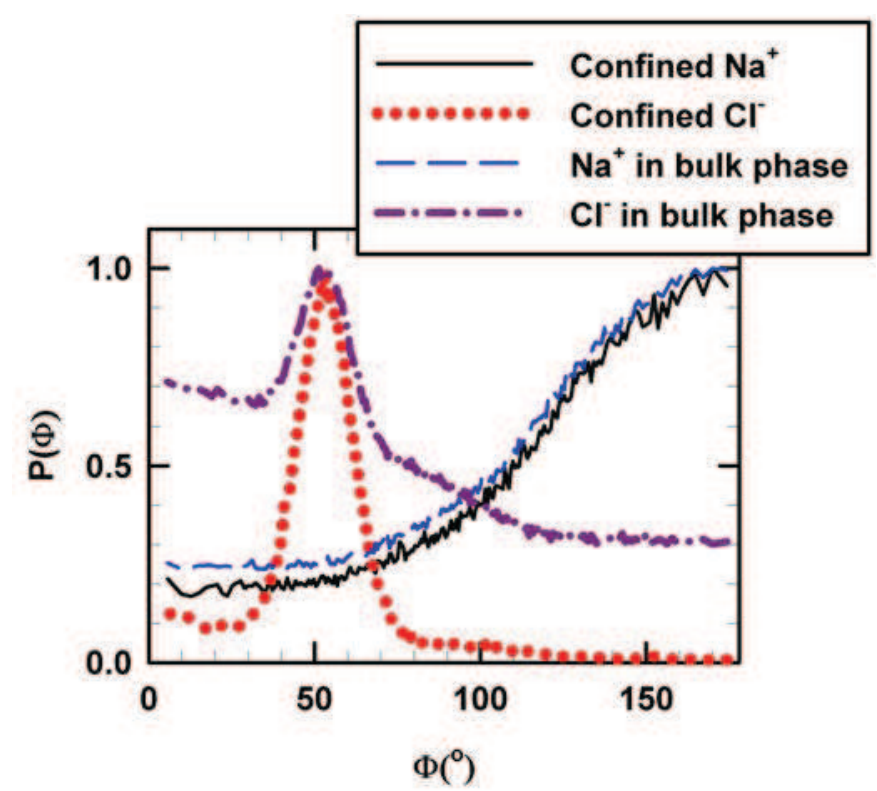

Fig. 6 Angular distribution between the dipole moment of water molecules and the vector between ions and the centre of mass of water molecules inside the nanocavity and in the bulk phase. 